\title{
Reply to "Serum high-sensitivity C-reactive protein in patients with obstructive sleep apnea with special reference to metabolic syndrome" by Kawada (Letter to the Editor)
}

\author{
Wei-Te $\mathrm{Wu}^{1} \cdot$ Saou-Hsing Liou ${ }^{1,2}$
}

Received: 3 August 2015 /Revised: 12 September 2015 / Accepted: 18 September 2015 /Published online: 28 September 2015

(C) Springer-Verlag Berlin Heidelberg 2015

\section{Dear editor,}

In our report, we used the AHI as both a continuous or categorical variable to do sensitivity analysis of how inputs and parameters affect outputs. The results showed that AHI (as a continuous variable) was significantly associated with hs-CRP (model 2, $\beta=0.125, p=0.009$ ) and also had a significant association with hs-CRP (model $2, \beta=0.533$, $p=0.005$ ) for severe OSA (as a categorical variable) when compared to the non-OSA group after being adjusting for age, smoking, drinking, and MetS status [1]. Based on the American Academy of Sleep Medicine (AASM) consensus report, the severity of OSA by classification of AHI is commonly used, such as severe OSA (AHI $>30 / \mathrm{h})$, moderate OSA (AHI, 15-30/h), persons at risk for OSA (AHI, $5-14 / \mathrm{h}$ ), and normal persons (AHI $<5 / \mathrm{h}$ ) [2]. Therefore, we believe that OSA classification can provide more detailed and useful information to readers. Similarly, the cut-point of hs-CRP as the value of $80^{\text {th }}$ percentile or $90^{\text {th }}$ percentile is also a sensitive analysis to confirm our results.

Saou-Hsing Liou

shliou@nhri.org.tw

1 National Institute of Environmental Health Sciences, National Health Research Institutes, 35 Keyan Road, ZhunanTown, Miaoli County 35053, Taiwan, Republic of China

2 Department of Public Health, National Defense Medical Center, Taipei, Taiwan
Our study shows that OSA was strongly associated with hsCRP and that the effect of OSA on CRP is independent of MetS. Indeed, results from this cross-sectional study may not be interpreted as having a causal association of MetS in the pathway from OSA to elevated hs-CRP. However, a previous a review article showed that OSA is thought possibly to lead to intermittent hypoxia, increased systemic inflammation that is promoted by upregulation of NF- $\mathrm{KB}$ leading to increased levels of TNF, and possibly by activation of HIF-1 as well as via increased levels of IL-6 and CRP, and thereby induce endothelial dysfunction [3]. Besides, we do not ignore the importance of obesity as a determinant of increased Hs-CRP. Thus, we concluded that CRP levels are elevated in OSA patients, and that this probably reflects both obesity and intermittent hypoxia.

Conflict of interest The authors declare that they have no competing interests.

Disclosure statement The authors have indicated no financial support.

\section{References}

1. Wu WT, Tsai SS, Shih TS, Lin MH, Chou TC, Ting H, Wu TN, Liou SH (2015). The impact of obstructive sleep apnea on high-sensitivity C-reactive protein in subjects with or without metabolic syndrome. Sleep Breath Apr 7. doi: 10.1007/s11325-015-1166-2

2. Ruehland WR, Rochford PD, O’Donoghue FJ, Pierce RJ, Singh P, Thornton AT (2009) The new AASM criteria for scoring hypopneas: impact on the apnea hypopnea index. Sleep 32:150-157

3. Kohler M, Stradling JR (2010) Mechanisms of vascular damage in obstructive sleep apnea. Nat Rev Cardiol 7:677-685 